\title{
Association of DNA methyltransferase polymorphisms with susceptibility to primary gouty arthritis
}

\author{
XIAOWU ZHONG ${ }^{1-3^{*}}$, YUANHONG PENG ${ }^{4 *}$, CHENGJIAO YAO $^{2 *}$, YUFENG QING $^{4}$, QIBIN YANG ${ }^{4}$, \\ XIAOLAN GUO ${ }^{1,3}$, WENGUANG XIE ${ }^{1,2}$, MINGCAI ZHAO ${ }^{1-3}$, XIAOMING CAI ${ }^{5}$ and JING-GUO ZHOU 3,4 \\ ${ }^{1}$ Department of Clinical Laboratory and ${ }^{2}$ Medicine Research Center, Affiliated Hospital of North Sichuan Medical College, \\ Nanchong, Sichuan 637000; ${ }^{3}$ Department of Laboratory Medicine, North Sichuan Medical College, Nanchong, \\ Sichuan 637007; ${ }^{4}$ Department of Rheumatology and Immunology of the Affiliated Hospital, \\ North Sichuan Medical College, Nanchong, Sichuan 637000; ${ }^{5}$ Department of Biology, \\ North Sichuan Medical College, Nanchong, Sichuan 637007, P.R. China
}

Received April 13, 2016; Accepted July 27, 2016

DOI: 10.3892/br.2016.746

\begin{abstract}
Gouty arthritis is the most common type of inflammatory and immune disease, and the prevalence and incidence of gout increases annually. Genetic variations in the DNA methyltransferases (DNMTs) gene have not, to the best of our knowledge, been reported to influence gene expression and to participate in the pathogenesis of gout. The aim of the present study was to investigate whether the DNMT1, DNMT3A and $D N M T 3 B$ polymorphisms contribute to gout susceptibility. These polymorphisms were screened for in 336 gout patients and 306 healthy control subjects (from a South China population) for association with gout. The distribution frequencies of DNMT1 rs2228611 AA genotype $(\mathrm{P}=0.007)$ and $\mathrm{A}$ allele $(\mathrm{P}=0.002$; odds ratio $=1.508,95 \%$ confidence interval=1.158-1.964) were found to be significantly increased in the gout patients when compared with those in the healthy control subjects. The rs1550117 in DNMT3A and rs2424913 in $D N M T 3 B$ exhibited no significant associations with gout susceptibility between the patients and control subjects. These results demonstrated that the DNMT1 rs2228611 polymorphism may be involved in the pathogenesis of gout, while DNMT3A rs1550117 and DNMT3B rs2424913 did not show any obvious significance in the current study; thus, may not be used as risk factors to predict the susceptibility to gout. However, further studies are required to investigate the functions and regulatory mechanism of the polymorphisms of DNMTs in gout.
\end{abstract}

Correspondence to: Professor Jing-Guo Zhou, Department of Rheumatology and Immunology of the Affiliated Hospital, North Sichuan Medical College, 63 Wenhua Road, Nanchong, Sichuan 637000, P.R. China

E-mail: jgzhou@nsmc.edu.cn

*Contributed equally

Key words: gouty arthritis, epigenetics, DNA methyltransferases, polymorphism

\section{Introduction}

Gout arthritis is the most common inflammatory and immune disease affecting 1-2\% of adults worldwide, which is associated with elevated serum urate levels and the deposition of monosodium urate (MSU) in the joints (1). Previous studies show that gout is also associated with the genetic background, a purine rich diet and alcohol consumption (2-4), and epidemiological studies have suggested that the prevalence and incidence of gout are increasing globally $(5,6)$. Over the past decade, significant scientific advances have been made in understanding the pathogenesis and treatment of gout. Being a complex disease, the identification of genetic and environmental risk factors for gout may facilitate with investigating the pathogenesis of gout. As with other diseases, epigenetic events or heritable changes in gene expression capacity without DNA sequence alterations may be evaluated to gain insight into the concrete pathogenesis of gout (7). DNA methylation is the most common epigenetic modification, and is important in transcription and the chromatin structure (8). The process of DNA methylation usually occurs at the $\mathrm{CpG}$ sites and the methyl group to the 5' position of a cytosine in a CpG dinucleotide conferred by DNA methyltransferases (DNMTs) (9). Three primary DNMTs, DNMT1, DNMT3A and DNMT3B, may influence global DNA methylation (10). In addition, DNMT1 is a primary enzyme for maintaining methylation patterns during DNA replication, whereas DNMT3A and DNMT3B act predominantly as the de novo methyltransferases, and create novel methylation patterns (11-13).

In addition, DNA methylation and its regulatory enzymes have been implicated in a diverse set of biological processes, including $\mathrm{X}$ chromosome inactivation, genomic imprinting, as well as autoimmunity (14-17). Mutation of the human DNMTs alters gene expression and may provide insight into the mechanism of various diseases, such as centromere instability, acute myeloid leukemia and immunodeficiency (18-20). Therefore, it DNMTs may be important in the pathogenesis of autoimmune diseases. The single nucleotide polymorphism (SNP) $14463 \mathrm{G}>\mathrm{C}$ of the DNMT1 gene was demonstrated to be associated with higher lupus disease activity (21). And Nam et al (22) 
identified that the $-283 \mathrm{C} / \mathrm{T}$ polymorphism at the promoter of the DNMT3B gene was associated with progression of joint destruction in rheumatoid arthritis (22). However, thus far, to the best of our knowledge, no studies have investigated the DNMT polymorphism association with gouty arthritis. In the present study, 336 primary gout patients and 306 healthy control subjects were employed to investigate the three SNPs of the DNMT gene and the susceptibility to primary gouty arthritis in a Chinese Han population, and aimed to gain a comprehensive understanding of their clinical relevance with regard to the development of gout.

\section{Materials and methods}

Subjects and ethical approval. All participants were recruited from the Department of Rheumatology, the Affiliated Hospital of North Sichuan Medical College (Nanchong, China) between February 2013 and April 2015. The study group consisted of 336 primary gouty arthritis patients and 306 healthy control subjects from a Chinese Han population. The gout patients were confirmed by a clinical endocrinology physician, according to the American College of Rheumatology classification criteria (23). The patients were not receiving any systemic anti-inflammatory treatment, or medication to control the production and elimination of uric acid prior to obtaining blood samples. The age-matched healthy control subjects, who had no history of gout or any systemic inflammatory disease, were enrolled in the present study. All the participants were of Chinese Han descent. Venous blood samples $(2 \mathrm{ml})$ from all participants were obtained in the morning, following an overnight fasting of at least $12 \mathrm{~h}$, and collected into sterile, single-use, anticoagulant-coated tubes and immediately sent to the laboratory for genetic testing. This study was approved by the Ethics Committee of the Affiliated Hospital of the North Sichuan Medical College. All participants provided written informed consent.

Clinical and laboratory examination. All of the clinical data and measurements were assessed and carefully recorded at the Department of Clinical Laboratory, the Affiliated Hospital of North Sichuan Medical College. The body mass index (BMI) was defined as weight in kilograms divided by the square of height in meters. The plasma biochemical values of all the individuals were measured using $7170 \mathrm{~S}$ Automatic Chemistry Analyzer (Hitachi Co., Ltd., Tokyo, Japan). In addition, the plasma hematological values were obtained according to routine laboratory testing methods. All measurements were performed by an expert who was blinded to the study.

Genomic DNA preparation. Genomic DNA was obtained using the TIANamp Blood DNA kit (Tiangen Biotech, Co., Ltd., Beijing, China) according to the manufacturer's instructions. DNA was isolated from the whole blood samples $(2 \mathrm{ml})$ of the 336 primary gout patients and 306 healthy control subjects in each collection tube, and eluted to a final elution volume of $50 \mu \mathrm{l}$ with Tris- $\mathrm{HCl}$ buffer (10 mmol/l; $\mathrm{pH} 8.0)$. The optical densities of the DNA samples were quantified using a NanoDrop 2000c spectrophotometer (Thermo Fisher Scientific, Inc., Wilmington, DE, USA) to determine the
DNA concentration. The DNA samples were stored at $-80^{\circ} \mathrm{C}$ until use.

Analysis of the DNMT polymorphisms. The rs2228611 in DNMT1, rs1550117 in DNMT3A and rs2424913 in DNMT3B were selected from the NCBI SNP Database (http://www. ncbi.nlm.nih.gov/snp/). The detailed information regarding these SNPs in DNMTs gene is presented in Table I. SNP genotyping was performed using a TaqMan real-time polymerase chain reaction (PCR) assay (Thermo Fisher Scientific, Inc., Waltham, MA, USA) according to the protocol described by Qing et al $(24,25)$. The TaqMan assays for the DNMT gene variants were designed using Primer Express software version 3.0 (Thermo Fisher Scientific, Inc.). The two probes labeled with a fluorescent reporter dye (VIC or FAM) and a non-fluorescing quencher/minor groove binder were used. During the primer extension phase of PCR, the 5' nuclease activity of the Taq polymerase cleaves and releases the fluorescence of the reporter dye. Upon termination of PCR, the samples were genotyped by analyzing the fluorescence of the two dyes on the ABI PRISM ${ }^{\circledR} 7900$ HT Sequence Detection System (Thermo Fisher Scientific, Inc.). TaqMan real-time PCR was performed according to the manufacturer's standard PCR protocol. The 5.0- $\mu$ l PCR reaction system was composed as follows: $1 \mathrm{ng}$ Total genomic DNA was mixed with the supplied 2X TaqMan Universal PCR Master Mix No AmpErase UNG and TaqMan Assay Mix to a final volume of $5.0 \mu \mathrm{l}$. The PCR reactions were performed in a 96-well plate on the ABI PRISM ${ }^{\circledR} 7900 H T$ Sequence Detection system (Thermo Fisher Scientific, Inc.). The PCR procedure was performed as follows: Initial denaturation at $95^{\circ} \mathrm{C}$ for $10 \mathrm{~min}$, amplification was conducted for 40 cycles at $92^{\circ} \mathrm{C}$ for $15 \mathrm{sec}$ and $60^{\circ} \mathrm{C}$ for $60 \mathrm{sec}$. The investigator who performed the genotyping was blinded to the patients' clinical status. Additionally, the DNA of the gout patients and healthy control subjects were genotyped together on the same plates with duplicates samples (15\%) to assess intraplate variation and interpolate genotype quality. No discrepancies in the genotypes were detected.

Statistical analysis. All statistical analysis was performed using SPSS statistical software, version 22.0 (IBM SPSS, Armonk, NY, USA). Student's t-test was performed to compare the clinical parameters between patients with gout and the healthy control subjects. Each DNMTs polymorphism was analyzed for deviations from the Hardy-Weinberg equilibrium (HWE) using $\chi^{2}$ test. And the $\chi^{2}$ analysis was used to compare the allele and genotype frequencies between the cases and control subjects. The odds ratios (ORs) and $95 \%$ confidence intervals (CIs) were calculated to assess the associations between genotypes and gout patients. Genotype and allele frequencies were performed using the SHEsis software (http://analysis.bio-X.cn/myAnalysis.php) (26). P<0.05 was considered to indicate a statistically significant difference.

\section{Results}

Subject characteristics. A total of 642 subjects were enrolled in the present study, including 306 healthy individuals and 336 patients with primary gout. The clinical characteristics 
Table I. SNPs from DNA methyltransferases.

\begin{tabular}{lclcccccc}
\hline Gene & SNP & Location & Chromosome & $\begin{array}{c}\text { Chromosome } \\
\text { position (bp) }\end{array}$ & From & To & Change & Codon \\
\hline DNMT1 & rs2228611 & Coding region & 19 & 10156401 & $\mathrm{G}$ & $\mathrm{A}$ & Pro463Pro & 463 \\
DNMT3A & rs1550117 & 5' near gene & 2 & 25343038 & $\mathrm{G}$ & $\mathrm{A}$ & $25565907 \mathrm{~A}>\mathrm{G}$ & - \\
DNMT3B & rs2424913 & Intron & 20 & 32786453 & $\mathrm{C}$ & $\mathrm{T}$ & $31374259 \mathrm{C}>\mathrm{T}$ & - \\
\hline
\end{tabular}

SNP, single nucleotide polymorphisms.

Table II. Characteristics of the study samples (mean \pm standard deviation).

\begin{tabular}{|c|c|c|c|}
\hline Characteristic & Gout $(n=336)$ & Control (n=306) & P-value \\
\hline Age (years) & $47.64 \pm 9.63$ & $46.02 \pm 7.99$ & $>0.05$ \\
\hline Body mass index $\left(\mathrm{kg} / \mathrm{m}^{2}\right)$ & $25.22 \pm 2.39$ & $23.02 \pm 2.14$ & $<0.01$ \\
\hline Serum uric acid $(\mu \mathrm{mol} / \mathrm{l})$ & $515.91 \pm 80.51$ & $324.60 \pm 34.59$ & $<0.01$ \\
\hline Monocyte count (x 109/1) & $0.66 \pm 0.20$ & $0.45 \pm 0.13$ & $<0.01$ \\
\hline Neutrophile granulocyte counts $\left(\mathrm{x} 10^{9} / 1\right)$ & $4.97 \pm 1.42$ & $3.26 \pm 0.56$ & $<0.01$ \\
\hline White blood cell count $\left(\times 10^{9} / 1\right)$ & $8.30 \pm 1.44$ & $6.16 \pm 0.99$ & $<0.01$ \\
\hline Lymphocyte count $\left(\times 10^{9} / 1\right)$ & $1.86 \pm 0.73$ & $1.82 \pm 0.56$ & $>0.05$ \\
\hline Serum glucose $(\mathrm{mmol} / \mathrm{l})$ & $6.07 \pm 0.90$ & $5.10 \pm 0.44$ & $<0.01$ \\
\hline High-density lipoprotein (mmol/l) & $1.09 \pm 0.34$ & $1.27 \pm 0.17$ & $<0.01$ \\
\hline Low-density lipoprotein (mmol/l) & $2.53 \pm 0.78$ & $2.80 \pm 0.39$ & $<0.01$ \\
\hline Very low-density lipoprotein (mmol/l) & $1.13 \pm 0.57$ & $0.55 \pm 0.23$ & $<0.01$ \\
\hline Triglycerides $(\mathrm{mmol} / \mathrm{l})$ & $2.12 \pm 1.15$ & $1.11 \pm 0.32$ & $<0.01$ \\
\hline Total cholesterol (mmol/l) & $4.67 \pm 0.92$ & $4.48 \pm 0.49$ & $>0.05$ \\
\hline
\end{tabular}

Data were compared using Student's t-test and $\mathrm{P}<0.05$ was considered to indicate a statistically significant difference.

and laboratory data of participants are presented in Table II. No significant differences were observed in age, lymphocyte count (LY) and total cholesterol (TC) between the patients and controls $(\mathrm{P}>0.05)$. The gout patients had significantly higher BMIs, serum uric acid (sUA), monocyte counts (MO), neutrophile granulocyte counts (GR), white blood cell counts (WBC), serum glucose (GLU), low-density lipoprotein (LDL-C), very low-density lipoprotein (VLDL) and triglycerides (TG) compared with the healthy control subjects $(\mathrm{P}<0.01)$. However, the levels of high-density lipoprotein (HDL-C) were markedly higher in healthy control subjects compared with the gout patients $(\mathrm{P}<0.01)$.

Associations between DNMT gene SNPs and gout risk. In the present study, three DNMT gene SNPs were obtained from the NCBI SNP database: The rs2228611 SNP, in the coding region of DNMT1, is located on chromosome 19, while the rs 1550117 is located near the $5^{\prime}$ region of DNMT3A on chromosome 2. The third SNP, rs2424913 was within the intron of DNMT3B on chromosome 20 (Table I). All the genotyped distributions were consistent with those expected from the HWE analysis between all investigated groups $(\mathrm{P}>0.05)$. As shown in Table III, the distribution frequencies of the rs2228611 AA genotype $\left(\chi^{2}=9.378 ; \mathrm{P}=0.007\right)$ and A allele $\left(\chi^{2}=9.772 ; \mathrm{P}=0.002\right.$ and $\mathrm{OR}=1.508,95 \% \mathrm{CI}=1.158-1.964)$ in the gout patients were significantly increased compared with the healthy control subjects, which indicated significant differences of the genotype and allele frequencies of rs2228611 in DNMT1 in patients as compared with the healthy control subjects.

In the control subjects, the frequencies of $\mathrm{GG}, \mathrm{AG}$ and AA genotypes, and $\mathrm{G}$ and A alleles of the DNMT3A gene rs1550117 were $0.680,0.307,0.013,0.833$ and 0.167 , respectively, while the frequencies of GG, AG and AA genotypes, and $\mathrm{G}$ and $\mathrm{A}$ alleles in the patients with gout were $0.652,0.330$, $0.018,0.817$ and 0.183 , respectively. In addition, the DNMT3B gene rs2424913 distributions of the genotype and allele did not show any associations with gout susceptibility between patients and control subjects $\left(\chi^{2}=1.312\right.$ and $1.266 ; \mathrm{P}=0.531$ and $0.252)$. Of the gout patients, $64(0.190)$ were heterozygous for CT, 8 (0.024) were homozygous for C and 264 (0.786) for T. In addition, the numbers and frequencies of $\mathrm{CC}, \mathrm{CT}$ and $\mathrm{TT}$ genotypes in the healthy control subjects were 9 (0.029), 68 (0.222) and 229 (0.748), respectively.

\section{Discussion}

Gout is a type of inflammatory arthritis caused by deposition of MSU crystals in the tissues and organs, and its prevalence and incidence has increased across various regions of the world in recent years (6). However, the precise pathogenesis of gout has 
Table III. Association analysis of DNMT1, DNMT3A and DNMT3B SNPs in gout.

\begin{tabular}{|c|c|c|c|c|c|}
\hline \multirow[b]{2}{*}{ Gene } & \multicolumn{2}{|c|}{ Cohort } & \multirow[b]{2}{*}{$\chi^{2}$} & \multirow[b]{2}{*}{ P-value } & \multirow[b]{2}{*}{ OR $(95 \% \mathrm{CI})$} \\
\hline & Patient & Control & & & \\
\hline \multicolumn{6}{|c|}{$D N M T 1$ rs2228611 } \\
\hline $\mathrm{G} / \mathrm{G}$ & $179(0.533)$ & $197(0.644)$ & & & \\
\hline $\mathrm{A} / \mathrm{G}$ & $136(0.405)$ & $100(0.327)$ & & & \\
\hline $\mathrm{A} / \mathrm{A}$ & $21(0.062)$ & $9(0.029)$ & 9.378 & 0.007 & \\
\hline G & $494(0.735)$ & $484(0.807)$ & & & \\
\hline A & $178(0.265)$ & $118(0.193)$ & 9.772 & 0.002 & $1.508(1.158-1.964)$ \\
\hline \multicolumn{6}{|c|}{ DNMT3A rs 1550117} \\
\hline $\mathrm{G} / \mathrm{G}$ & $219(0.652)$ & $208(0.680)$ & & & \\
\hline $\mathrm{A} / \mathrm{G}$ & $111(0.330)$ & $94(0.307)$ & & & \\
\hline $\mathrm{A} / \mathrm{A}$ & $6(0.018)$ & $4(0.013)$ & 0.594 & 0.707 & \\
\hline G & $549(0.817)$ & $510(0.833)$ & & & \\
\hline A & $123(0.183)$ & $102(0.167)$ & 0.692 & 0.441 & $0.893(0.669-1.192)$ \\
\hline \multicolumn{6}{|c|}{ DNMT3B rs2424913 } \\
\hline $\mathrm{C} / \mathrm{C}$ & $8(0.024)$ & $9(0.029)$ & & & \\
\hline $\mathrm{C} / \mathrm{T}$ & $64(0.190)$ & $68(0.222)$ & & & \\
\hline $\mathrm{T} / \mathrm{T}$ & $264(0.786)$ & $229(0.748)$ & 1.312 & 0.531 & \\
\hline $\mathrm{C}$ & $80(0.119)$ & $86(0.141)$ & & & \\
\hline $\mathrm{T}$ & $592(0.881)$ & $526(0.859)$ & 1.266 & 0.252 & $1.210(0.873-1.677)$ \\
\hline
\end{tabular}

SNP, single nucleotide polymorphism; OR, odds ratio; CI, confidence interval.

not been clarified. Therefore, further studies investigating gout will facilitate a better understanding of its pathogenesis and treatment. In the present study, the concentration of sUA, MO, GR, WBC, GLU, LDL-C, VLDL and TG were identified to be significantly higher in the gout patients compared with those in the healthy control subjects, which indicates that a close association between sUA levels and gout exists. Certain studies have demonstrated that genetic variation of ATP binding cassette subfamily $\mathrm{G}$ member $2(A B C G 2)$, solute carrier family 2 member 9 (SLC2A9), and solute carrier family 22 member 12 (SLC22A12) genes are involved in control of sUA levels, and the SNPs of $A B C G 2, S L C 2 A 9$ and $S L C 22 A 12$ genes may increase the risk for an individual to develop gout (27-29). However, only $\sim 10 \%$ of patients with hyperuricaemia would eventually develop gout (30) while the remaining $90 \%$ patients would not, which prompts investigation of the molecular basis involved in the metabolism and regulation of urate to further reveal the pathogenesis of gout.

With increasing evidence suggesting that the epigenetic processes may contribute to promoting cancer and autoimmunity, DNMT gene polymorphisms were associated with various types of cancer and autoimmune diseases in different populations worldwide $(31,32)$. DNMT1 rs2228611 SNPs were associated with increased susceptibility to developing ovarian cancer, while there were no significant associations identified between rs2228611 and gastric cancer risk in the cases and controls $(33,34)$. Previous studies of DNMT3A rs1550117 demonstrated that the SNPs may modulate the DNMT3A gene expression and contribute to genetic susceptibility to gastric and colorectal cancer development $(35,36)$. The rs 2424913 of the DNMT3B gene are significantly associated with a negative risk of colorectal cancer development in the African population (37). Although the genotype distribution of $D N M T 3 B$ rs2424913 demonstrated no significant differences between the children with immune thrombocytopenia (ITP) and the healthy individuals, the frequencies of the $\mathrm{T}$ allele were significantly increased in children with ITP (38). Conversely, Piotrowski et al (39) identified that the rs1550117 of DNMT3A exhibited a significant association with SLE in a Polish population when compared with healthy individuals, which indicated that this SNP may protect against SLE development. Recently, using Gene Expression Microarrays, DNMT gene (DNMT1, DNMT3A and DNMT3B) expression was demonstrated to be markedly different in patients with gout and in healthy control subjects (data not shown), which indicated that DNMT1, DNMT3A and DNMT3B may be associated with the risk of gout. To determine the association between DNMT genes and gout, the distribution of the polymorphisms between gout patients and healthy control subjects from a Chinese Han population were detected. The distribution frequencies of rs2228611 AA genotype and A allele were identified to be significantly increased in gout patients compared with healthy control subjects, which suggest that the AA genotype and A allele of rs2228611 were associated with the risk of gout in the Han Chinese population. To the best of our knowledge, this study is the first to investigate on the association of common SNPs in the DNMT1 gene and risk of gout in a Chinese Han population. Therefore, it is hypothesized that the DNMT1 rs2228611 polymorphism may be important in modulating DNMT1 gene expression, 
causing a genome-wide alteration of DNA methylation status, and influencing the transcription of other genes. Furthermore, the DNMT1 rs2228611 polymorphism may contribute to susceptibility to gout development. However, the functional effects of the DNMT1 rs2228611 polymorphism require further investigation.

In the present study, whether rs1550117 in the DNMT3A gene and rs2424913 in the DNMT3B gene are considered to be genetic susceptibility factors of gout was evaluated. However, no evidence for the involvement of DNMT3A rs1550117 and DNMT3B rs2424913 polymorphisms in susceptibility to gout was identified. Furthermore, the genotypes of these two polymorphisms demonstrated a similar distribution trend between the gout patients and the healthy control subjects in the Chinese Han population, which indicates that DNMT3A rs1550117 and $D N M T 3 B$ rs2424913 may not be used to evaluate susceptibility to gout.

In conclusion, the association between DNMT gene polymorphisms and gout susceptibility remains unknown. In the present study, DNMT1 rs2228611, DNMT3A rs1550117 and DNMT3B rs2424913 were investigated in Chinese Han patients with gout using TaqMan technology. The results demonstrated that the DNMT1 rs2228611 polymorphism may be an important genetic risk factor for the development of gout, while DNMT3A rs1550117 and DNMT3B rs2424913 did not appear to predict a susceptibility to gout. Multicenter studies are required to investigate the present findings further and the underlying biological function of these genes requires further investigation.

\section{Acknowledgements}

The present study was supported by a grant from the National Natural Science Foundation (grant nos. 81272047 and 81401767), the Science and Technology Support Program of Nanchong (grant no. 14A0061), the Scientific Research Fund of Sichuan Provincial Education Department (grant no. 15ZB0197), and the Doctoral Scientific Research Foundation of Research and Development Program of North Sichuan Medical College (grant no. CBY14-QD-07).

\section{References}

1. Richette P and Bardin T: Gout. Lancet 375: 318-328, 2010.

2. White JS: Comment on: New insights into the epidemiology of gout. Rheumatology (Oxford) 49: 613-614, author reply 614, 2010.

3. Wang M, Jiang X, Wu W and Zhang D: A meta-analysis of alcohol consumption and the risk of gout. Clin Rheumatol 32: $1641-1648,2013$.

4. Merriman TR, Choi HK and Dalbeth N: The genetic basis of gout. Rheum Dis Clin North Am 40: 279-290, 2014

5. Zhu Y, Pandya BJ and Choi HK: Prevalence of gout and hyperuricemia in the US general population: The National Health and Nutrition Examination Survey 2007-2008. Arthritis Rheum 63: 3136-3141, 2011.

6. Kuo CF, Grainge MJ, Zhang W and Doherty M: Global epidemiology of gout: prevalence, incidence and risk factors. Nat Rev Rheumatol 11: 649-662, 2015

7. Khalil AM and Wahlestedt C: Epigenetic mechanisms of gene regulation during mammalian spermatogenesis. Epigenetics 3 : 21-28, 2008.

8. Jin B, Li Y and Robertson KD: DNA methylation: superior or subordinate in the epigenetic hierarchy? Genes Cancer 2 607-617, 2011

9. Bheemanaik S, Reddy YV and Rao DN: Structure, function and mechanism of exocyclic DNA methyltransferases. Biochem J 399: 177-190, 2006
10. Moore LD, Le T and Fan G: DNA methylation and its basic function. Neuropsychopharmacology 38: 23-38, 2013.

11. Okano M, Bell DW, Haber DA and Li E: DNA methyltransferases Dnmt3a and Dnmt3b are essential for de novo methylation and mammalian development. Cell 99: 247-257, 1999.

12. Probst AV, Dunleavy E and Almouzni G: Epigenetic inheritance during the cell cycle. Nat Rev Mol Cell Biol 10: 192-206, 2009.

13. Chédin F: The DNMT3 family of mammalian de novo DNA methyltransferases. Prog Mol Biol Transl Sci 101: 255-285, 2011.

14. Basu R and Zhang LF: X chromosome inactivation: a silence that needs to be broken. Genesis 49: 821-834, 2011.

15. Sharp AJ, Stathaki E, Migliavacca E, Brahmachary M, Montgomery SB, Dupre Y and Antonarakis SE: DNA methylation profiles of human active and inactive $\mathrm{X}$ chromosomes. Genome Res 21: 1592-1600, 2011.

16. Ferguson-Smith AC: Genomic imprinting: the emergence of an epigenetic paradigm. Nat Rev Genet 12: 565-575, 2011.

17. Richardson B: DNA methylation and autoimmune disease. Clin Immunol 109: 72-79, 2003.

18. Conway O'Brien E, Prideaux S and Chevassut T: The epigenetic landscape of acute myeloid leukemia. Adv Hematol 2014: 103175, 2014

19. Jost E, Lin Q, Weidner CI, Wilop S, Hoffmann M, Walenda T, Schemionek M, Herrmann O, Zenke M, Brümmendorf TH, et al: Epimutations mimic genomic mutations of DNMT3A in acute myeloid leukemia. Leukemia 28: 1227-1234, 2014.

20. Hansen RS, Wijmenga C, Luo P, Stanek AM, Canfield TK, Weemaes CM and Gartler SM: The DNMT3B DNA methyltransferase gene is mutated in the ICF immunodeficiency syndrome. Proc Natl Acad Sci USA 96: 14412-14417, 1999.

21. Park BL, Kim LH, Shin HD, Park YW, Uhm WS and Bae SC: Association analyses of DNA methyltransferase-1 (DNMT1) polymorphisms with systemic lupus erythematosus. J Hum Genet 49: 642-646, 2004

22. Nam EJ, Kim KH, Han SW, Cho CM, Lee J, Park JY and Kang YM: The $-283 \mathrm{C} / \mathrm{T}$ polymorphism of the DNMT3B gene influences the progression of joint destruction in rheumatoid arthritis. Rheumatol Int 30: 1299-1303, 2010.

23. Wallace SL, Robinson H, Masi AT, Decker JL, McCarty DJ and Yü TF: Preliminary criteria for the classification of the acute arthritis of primary gout. Arthritis Rheum 20: 895-900, 1977.

24. Qing YF, Zhou JG, Li M, Xie WG, Huang CP, Zeng SP and Yin L: No evidence for involvement of the toll-like receptor (TLR) 4 gene Asp299Gly and Thr399Ile polymorphisms in susceptibility to primary gouty arthritis. Rheumatol Int 33: 2937-2941, 2013.

25. Qing YF, Zhou JG, Zhang QB, Wang DS, Li M, Yang QB, Huang CP, Yin L, Pan SY, Xie WG, et al: Association of TLR4 Gene rs2149356 polymorphism with primary gouty arthritis in a case-control study. PLoS One 8: e64845, 2013.

26. Shi YY and He L: SHEsis, a powerful software platform for analyses of linkage disequilibrium, haplotype construction, and genetic association at polymorphism loci. Cell Res 15: 97-98, 2005.

27. Kolz M, Johnson T, Sanna S, Teumer A, Vitart V, Perola M, Mangino M, Albrecht E, Wallace C, Farrall M, et al; EUROSPAN Consortium; ENGAGE Consortium; PROCARDIS Consortium; KORA Study; WTCCC: Meta-analysis of 28,141 individuals identifies common variants within five new loci that influence uric acid concentrations. PLoS Genet 5: e1000504, 2009.

28. Matsuo H, Nakayama A, Sakiyama M, Chiba T, Shimizu S, Kawamura Y, Nakashima H, Nakamura T, Takada Y, Oikawa Y, et al: ABCG2 dysfunction causes hyperuricemia due to both renal urate underexcretion and renal urate overload. Sci Rep 4: 3755, 2014.

29. Zhou D, Liu Y, Zhang X, Gu X, Wang H, Luo X, Zhang J, Zou H and Guan M: Functional polymorphisms of the $A B C G 2$ gene are associated with gout disease in the Chinese Han male population. Int J Mol Sci 15: 9149-9159, 2014.

30. Bardin T and Richette P: Definition of hyperuricemia and gouty conditions. Curr Opin Rheumatol 26: 186-191, 2014.

31. Sharma S, Kelly TK and Jones PA: Epigenetics in cancer. Carcinogenesis 31: 27-36, 2010.

32. Meda F, Folci M, Baccarelli A and Selmi C: The epigenetics of autoimmunity. Cell Mol Immunol 8: 226-236, 2011.

33. Mostowska A, Sajdak S, Pawlik P, Lianeri M and Jagodzinski PP: DNMT1, DNMT3A and DNMT3B gene variants in relation to ovarian cancer risk in the Polish population. Mol Biol Rep 40: 4893-4899, 2013 
34. Yang XX, He XQ, Li FX, Wu YS, Gao $\mathrm{Y}$ and $\mathrm{Li} \mathrm{M}$ Risk-association of DNA methyltransferases polymorphisms with gastric cancer in the Southern Chinese population. Int J Mol Sci 13: 8364-8378, 2012.

35. Fan H, Liu D, Qiu X, Qiao F, Wu Q, Su X, Zhang F, Song Y, Zhao Z and Xie W: A functional polymorphism in the DNA methyltransferase-3A promoter modifies the susceptibility in gastric cancer but not in esophageal carcinoma. BMC Med 8: $12,2010$.

36. Zhao Z, Li C, Song Y, Wu Q, Qiao F and Fan H: Association of the DNMT3A $-448 \mathrm{~A}>\mathrm{G}$ polymorphism with genetic susceptibility to colorectal cancer. Oncol Lett 3: 450-454, 2012.
37. Duan F, Cui S, Song C, Dai L, Zhao X and Zhang X: Systematic evaluation of cancer risk associated with DNMT3B polymorphisms. J Cancer Res Clin Oncol 141: 1205-1220, 2015.

38. Pesmatzoglou M, Lourou M, Goulielmos GN and Stiakaki E: DNA methyltransferase 3B gene promoter and interleukin-1 receptor antagonist polymorphisms in childhood immune thrombocytopenia. Clin Dev Immunol 2012: 352059, 2012.

39. Piotrowski P, Grobelna MK, Wudarski M, Olesinska M and Jagodzinski PP: Genetic variants of DNMT3A and systemic lupus erythematosus susceptibility. Mod Rheumatol 25: 96-99, 2015. 